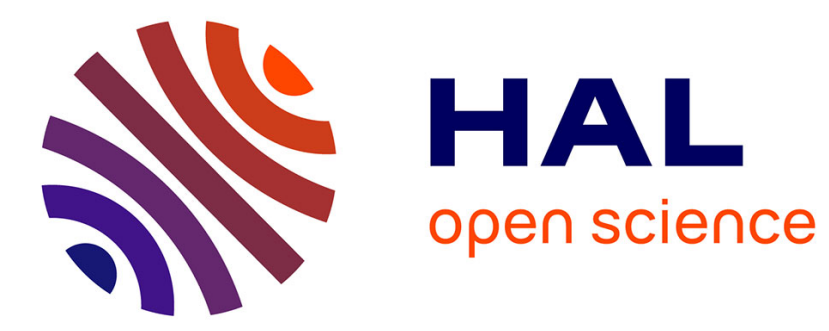

\title{
Spiral vortex breakdown as a global mode
}

F. Gallaire, M. Ruith, E. Meiburg, Jean-Marc Chomaz, Patrick Huerre

\section{To cite this version:}

F. Gallaire, M. Ruith, E. Meiburg, Jean-Marc Chomaz, Patrick Huerre. Spiral vortex breakdown as a global mode. Journal of Fluid Mechanics, 2006, 549 (february), pp.71-80. $10.1017 / \mathrm{s} 0022112005007834$. hal-01023370

\section{HAL Id: hal-01023370 \\ https://hal-polytechnique.archives-ouvertes.fr/hal-01023370}

Submitted on 20 Jul 2014

HAL is a multi-disciplinary open access archive for the deposit and dissemination of scientific research documents, whether they are published or not. The documents may come from teaching and research institutions in France or abroad, or from public or private research centers.
L'archive ouverte pluridisciplinaire HAL, est destinée au dépôt et à la diffusion de documents scientifiques de niveau recherche, publiés ou non, émanant des établissements d'enseignement et de recherche français ou étrangers, des laboratoires publics ou privés. 


\title{
Spiral vortex breakdown as a global mode
}

\author{
By FRANÇOIS GALLAIRE ${ }^{1}$, MICHAEL RUITH ${ }^{2}$, \\ ECKART MEIBUR G ${ }^{2}$, JEAN-MAR C CHOMAZ Z $^{3}$ \\ AND PATRICK HUERRE ${ }^{3}$ \\ ${ }^{1}$ Laboratoire J.-A. Dieudonné, CNRS, Université de Nice - Sophia Antipolis, Parc Valrose, F-06108
Nice, France \\ ${ }^{2}$ Department of Mechanical and Environmental Engineering, UCSB, Santa Barbara, CA 93106, USA \\ ${ }^{3}$ Laboratoire d'Hydrodynamique - LadHyX, CNRS - Ecole Polytechnique, F-91128 Palaiseau, France
}

(Received 21 July 2005 and in revised form 4 November 2005)

The spiral form of vortex breakdown observed in the numerical simulations of Ruith et al. (J. Fluid Mech., vol. 486, 2003, p. 331) is interpreted as a nonlinear global mode originating at the convective/absolute instability transition point in the lee of the vortex breakdown bubble. The local absolute frequency at the transition station is shown to yield a satisfactory prediction of the precession frequency measured in the three-dimensional direct numerical simulations.

\section{Introduction}

Vortex breakdown is a widespread phenomenon affecting swirling jets as soon as the swirl parameter $S$, which compares the intensity of the azimuthal velocity component to its axial counterpart, is large enough. It is observed both in its axisymmetric form, the so-called breakdown bubble, and in its spiral form. Spiral vortex breakdown appears to have been first described by Lambourne \& Bryer (1961) on delta-wings, Sarpkaya (1971), Faler \& Leibovich (1977) and Escudier \& Zehnder (1982) in tube experiments, and Spall \& Gatski (1991) in numerical simulations of the NavierStokes equations. The coexistence of bubble and spiral breakdown states for the same parameter settings was first visualized in the famous experiment of Lambourne \& Bryer (1961). The particle tracking velocimetry (PTV) measurements of Brücker \& Althaus (1995) have further revealed a periodic switch between the bubble and spiral states. The physical mechanisms at the origin of the helical forms of vortex breakdown have been actively discussed to a point where some authors have even questioned their existence and viewed them as pure visualization artifacts (see the review by Escudier 1988). We adopt here the point of view of Escudier (1988) and denote as spiral vortex breakdown the synchronized helical states characterized by an azimuthal wavenumbers of $m=1$, rotating in time in the same direction as the swirling base flow but winding in space in the opposite direction. These modes should be distinguished from higher-order helical co-winding modes that have also been observed for swirl numbers below vortex breakdown onset for instance by Escudier \& Zehnder (1982) or Billant, Chomaz \& Huerre (1998).

The aim of the present study is to demonstrate that the non-axisymmetric spiral vortex breakdown states observed at moderate Reynolds numbers may be interpreted as resulting from a global instability of the axisymmetric breakdown state. More specifically, following suggestions of Escudier, Bornstein \& Maxworthy (1982), Maxworthy (personal communication) and Delbende, Chomaz \& Huerre (1998), 
we examine whether the spiral vortex breakdown might result from the development of a nonlinear global mode triggered by the absolute instability of axisymmetric vortex breakdown viewed as an appropriate base flow. For a review of these concepts, the reader is referred to Huerre \& Monkewitz (1990) and Chomaz (2005). Yin et al. (2000) and Ruith et al. (2003) have recently shown that the absolute/convective instability properties associated with the $m=1$ mode of the Batchelor vortex compare well qualitatively with the experimental and numerical (respectively) characteristics of spiral vortex breakdown states. It should be stressed however that their swirling jet velocity profiles at various streamwise stations do not resemble those of the Batchelor vortex. Our objective is to quantitatively compare the predictive criterion of the weakly non-parallel nonlinear global mode theory of Couairon \& Chomaz (1999) and Pier, Huerre \& Chomaz (2001) to the three-dimensional numerical experiments of Ruith et al. (2003) using as a base flow their axisymmetric vortex breakdown states.

\section{Base flow and direct numerical simulation results}

The base flows are obtained by solving the axisymmetric Navier-Stokes equations in a semi-infinite domain as discussed in detail in Ruith et al. (2003). The velocity profile of Grabowski \& Berger (1976) is imposed at the inlet. If the centreline jet velocity $U_{c}$ and jet radius $R$ are used as reference scales the non-dimensional azimuthal, radial and axial velocities are

$$
\begin{array}{ll}
U_{\theta}(r, z=0)=S r\left(2-r^{2}\right) & \text { for } 0 \leqslant r \leqslant 1, \\
U_{\theta}(r, z=0)=S / r & \text { for } r>1, \\
U_{r}(r, z=0)=0, & \\
U_{z}(r, z=0)=1, &
\end{array}
$$

where the swirl number $S$ is the non-dimensional axial vorticity on the axis. The associated Reynolds number is defined as $R e=U_{c} R / v$, where $v$ is the kinematic viscosity.

A typical result of the axisymmetric direct numerical simulation (DNS) is depicted in figure 1(a) for $R e=200$ and $S=1$, and denoted as our reference case. This flow state is characterized by a vortex breakdown bubble with its associated recirculation zone and stagnation point. For the same parameter setting as in figure 1(a) but upon relaxing the axisymmetry assumption, one obtains the typical instantaneous streaklines illustrated in figure $1(b)$. The three-dimensional DNS was initiated by imposing an initial random noise on the base flow. According to Ruith et al. (2003), the flow settles into limit-cycle oscillations at a well-defined non-dimensional frequency $\omega_{G}^{N L}=1.18$.

\section{Local stability analysis}

The local stability analysis refers to the characteristics of the base flow at each axial station $z$, under the assumption of strict parallelism. The aim of the local study is to compute the maximum temporal growth rate $\omega_{i}^{\max }(z)$ as well as the local complex absolute frequency $\omega_{0}(z)$. The sign of its imaginary part $\omega_{0, i}(z)$ determines if the flow is locally absolutely unstable $\left(\omega_{0, i}(z)>0\right)$ or convectively unstable $\left(\omega_{0, i}(z)<0\right)$. The weakly non-parallel but strongly nonlinear theory of Pier et al. (2001) suggests that, if there exists a station $z_{C / A}$ where $\omega_{0, i}(z)$ vanishes and where the flow changes from convectively unstable for $z<z_{C / A}$ to absolutely unstable for $z>z_{C / A}$, a nonlinear global mode may be triggered with a front located at $z_{C / A}$. The global mode then inherits the real absolute frequency $\omega_{0}\left(z_{C / A}\right)$ as global frequency $\omega_{G}^{N L}$. 

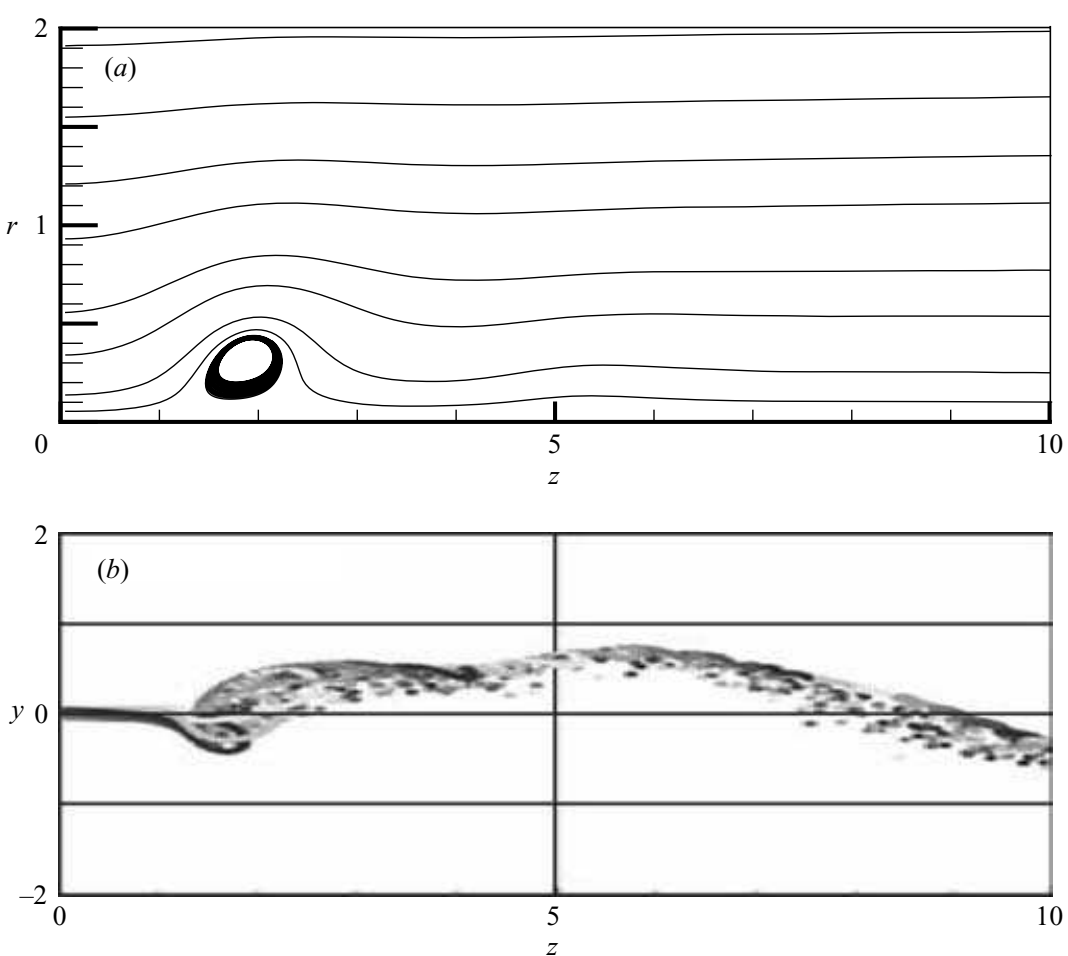

Figure 1. (a) Base flow streamlines at $R e=200, S=1$, obtained from DNS by imposing axisymmetry. $(b)$ Corresponding instantaneous streaklines in a $(y, z)$ projection at $t=1850$ for the same parameter setting (Ruith et al. 2003).

At a given station $z$ the base flow profiles $U_{z}(z, r), U_{\theta}(z, r)$ are first extracted from the axisymmetric DNS of Ruith et al. (2003) and interpolated onto a square Cartesian grid in order to match the input requirements of the stability code detailed below. Under the parallel flow hypothesis, the basic flow is assumed to be steady and uniform in the axial direction, with viscous diffusion being neglected. The radial velocity component $U_{r}(z, r)$ is also ignored. The validity of these assumptions may be quantified by evaluating the non-parallelism parameters

$$
\beta_{1}(z)=\sup _{r}\left(\frac{\partial U_{\theta}}{\partial z}, \frac{\partial U_{z}}{\partial z}\right), \quad \beta_{2}(z)=\sup _{r}\left(\frac{U_{r}}{\max \left(U_{z}, U_{\theta}\right)}\right) .
$$

Figure 2 represents the evolution of $\beta_{1}(z)$ and $\beta_{2}(z)$ as a function of the streamwise coordinate $z$. It is seen that the quasi-parallel assumption is mostly violated in the recirculation zone.

\subsection{Numerical implementation}

The spatio-temporal instability properties are retrieved from DNS of the linear impulse response originating from an initial localized disturbance as introduced by Delbende et al., (1998). The main feature of the method is that it directly gives access to the most amplified mode for each pair of ray velocity $v_{g}=z / t$ and azimuthal wavenumber $m$, without necessitating a complex-plane analysis. We focus our attention on the case $m=1$ and $v_{g}=0$ if not explicitly mentioned otherwise. Note that this procedure also allows one to efficiently access the temporally most amplified mode for each axial and azimuthal wavenumber pair $k$ and $m$. The Navier-Stokes 


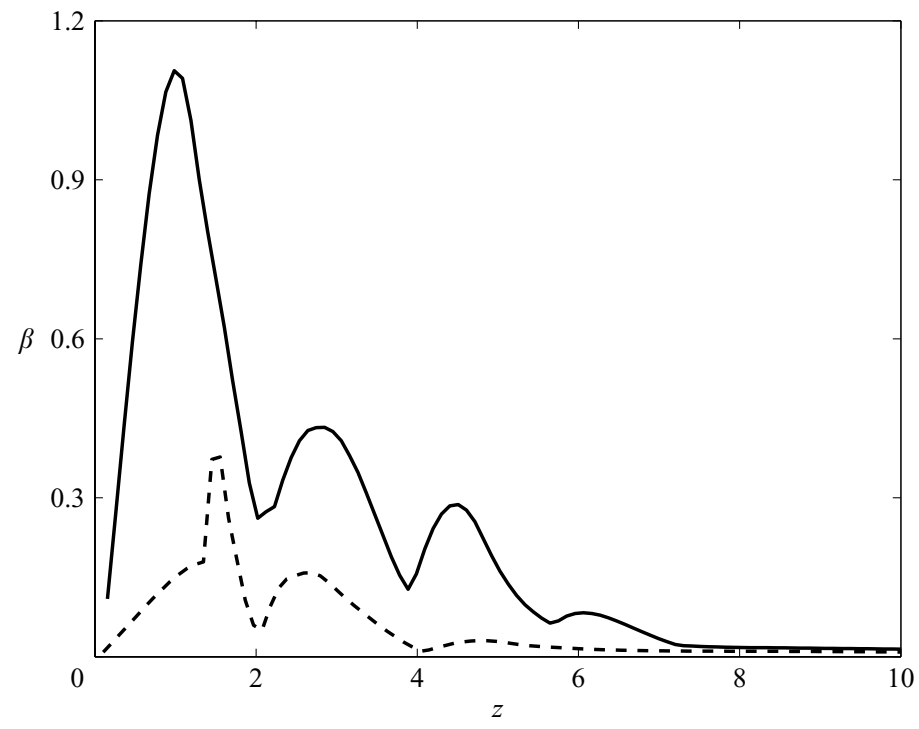

FIGURE 2. Evolution of the non-parallelism parameters $\beta_{1}(z)$ (solid line) and $\beta_{2}(z)$ (dashed line), as defined by equations $(3.1 a, b)$, as a function of $z$.

equations linearized around the parallel base flow under consideration at station $z$ are projected onto $1440 \times 128 \times 128$ Fourier modes along the three Cartesian directions. Periodicity is assumed in all directions $(\mathrm{Oz}$ is the homogeneous streamwise direction of the jet axis and $O x$ and $O y$ two arbitrary perpendicular directions $\dagger$ ). In physical space, this corresponds to a cubic mesh of spacing $\delta x=\delta y=\delta z=0.05$ and box lengths $L_{x}=L_{y}=6.4$, large enough for confinement due to periodicity in the $x$ and $y$-directions to be weak, and $L_{z}=72$, guaranteeing that the leading edge of the wave packet does not catch up with its trailing end. The time step $\delta t=0.005$ is chosen so as to satisfy a CFL-type numerical stability condition $\delta t / \delta z=0.1$ to be compared with the value of about 0.5 , charateristic of the inverse of the maximum velocity magnitude. Convergence has been satisfactorily checked by performing a highresolution simulation with $2160 \times 196 \times 196$ mesh points and $\delta x=\delta y=\delta z=0.033$.

In order to mimic the Dirac delta-function forcing in space and time, a divergencefree initial Gaussian perturbation of characteristic velocity perturbation amplitude 0.1 contained within a sphere of radius $\rho=0.5$ is imposed. As in Delbende et al., (1998), the impulse is centred at $z_{0}=15, r_{0}=1$ and $\theta=15^{\circ}$.

The impulse response is analysed by choosing as state variable the axial velocity perturbation $u_{z}(x, y, z, t)$ which is determined at the nodes of a cylindrical grid $u_{z}(r, \theta, z, t)$. The cylindrical coordinate space is discretized into 40 points in the radial direction in the range $0 \leqslant r \leqslant R_{\max }=L_{y}$ and 32 points in the azimuthal direction. The implied azimuthal wavenumber range restriction $|m|<16$ does not introduce any aliasing error since the neglected modes are all stable for the flows under consideration.

\subsection{Diagnostic tools}

In the laboratory frame, the development of the wave packet has to be considered along the specific ray $\left(z-z_{0}\right) / t=0$. Let $u_{z}(r, m, z, t)$ denote the azimuthal Fourier

$\dagger$ The homogeneous streamwise coordinate $z$ in the local instability study should not be mixed up with its inhomogeneous counterpart associated with the base flow. 
transform of $u_{z}(r, \theta, z, t)$. According to steepest-descent arguments (Bers 1983), the long-time behaviour of the impulse response along the ray $\left(z-z_{0}\right) / t=v_{g}$ is dominated by the mode with highest spatio-temporal growth rate according to the formula

$$
u_{r}(r, m, z, t) \propto g_{0}(r, m) t^{-1 / 2} \exp \left[k_{0}(m)\left(z-z_{0}\right)-\omega_{0}(m) t\right], t \rightarrow \infty,
$$

where $g_{0}(r, m)$ is the eigenfunction and $k_{0}(m)$ and $\omega_{0}(m)$ represent the complex absolute wavenumber and frequency observed in the laboratory frame. Following and adapting Delbende et al.'s methodology, one first recovers the amplitude and phase

$$
\begin{gathered}
A_{m}(z, t)=\left(\int_{0}^{R_{\max }}\left|u_{z}(r, m, z, t)\right|^{2} r \mathrm{~d} r\right)^{1 / 2}, \\
\phi_{m}(z, t)=\arg \left(u_{z}\left(r_{0}, m, z, t\right)\right), \text { with } 0 \leqslant \phi_{m}(k, t)<2 \pi .
\end{gathered}
$$

The absolute growth rate $\omega_{0, i}(m)$ and frequency $\omega_{0, r}(m)$ are then calculated via the formulae

$$
\begin{gathered}
\omega_{0, i}(m) \sim \frac{\ln \left(A_{m}\left(z_{0}, t_{2}\right) / A_{m}\left(z_{0}, t_{1}\right)\right)}{t_{2}-t_{1}}+\frac{\ln \left(t_{2}-t_{1}\right)}{2\left(t_{2}-t_{1}\right)}, \\
\omega_{0, r}(m) \sim-\frac{\phi_{m}\left(z_{0}, t_{3}\right)-\phi_{m}\left(z_{0}, t_{2}\right)}{t_{3}-t_{2}},
\end{gathered}
$$

where $t_{1}, t_{2}$ and $t_{3}$ are suitably selected as discussed below. Similarly, the real and imaginary parts of the absolute wavenumber observed at $z / t=0$ are retrieved according to

$$
\begin{gathered}
k_{0, r}(m) \sim \frac{\phi_{m}\left(z_{0}+\delta z, t_{2}\right)-\phi_{m}\left(z_{0}, t_{2}\right)}{\delta z}, \\
k_{0, i}(m) \sim-\ln \left(\frac{A_{m}\left(z_{0}+\delta z, t_{2}\right) A_{m}\left(z_{0}, t_{1}\right)}{A_{m}\left(z_{0}, t_{2}\right) A_{m}\left(z_{0}+\delta z t_{1} / t_{2}, t_{1}\right)}\right) \frac{t_{2}}{\delta z\left(t_{2}-t_{1}\right)} .
\end{gathered}
$$

A demodulation procedure based on both the Hilbert transform as in Delbende et al., (1998) and on the usual azimuthal Fourier transform $u_{z}(z, r, m, t)$ have been implemented to determine $A_{m}(z, t)$ and $\phi_{m}(z, t)$ given by (3.3)-(3.4). As mentioned in Gallaire \& Chomaz (2003), the Hilbert transform degrades the tails of the wave packet considerably when its spectrum does not vanish at $k=0$. As a result, the determination of $\omega_{0, i}$ when it is close to zero becomes less accurate. The second procedure, however, also has a shortcoming: since the signal is not demodulated, growth rates and other quantities may oscillate in time and space due to phase variations.

As noted by Delbende et al., (1998), the times $t_{1}$ and $t_{2}$ and the time-increment $t_{2}-t_{1}$ selected to numerically evaluate (3.5), (3.7) and (3.8) have to be large enough in order to ensure convergence towards the leading most-unstable mode and circumvent lowfrequency oscillations. On the other hand, in order to compute the mode frequency (3.6), the time interval $t_{3}-t_{2}$ has to be short enough so as to circumvent the difficulties associated with the discontinuous nature of the phase function $\phi_{m}(k, t)$ whenever it reaches 0 or $2 \pi$. In the present study, growth rates are calculated as follows: a sequence of times $\tau_{n}=\tau_{0}+n \tau$ with $\tau_{0}=9$ and $\tau=1.5$ is first chosen. Formulae (3.5)-(3.6) are then applied to successive time intervals in the sequence, with $t_{1}=\tau_{n-1}, t_{2}=\tau_{n}$ or $\tau_{n+1}$ or $\tau_{n+2}$ and $t_{3}=\tau_{n}+\delta \tau$ with $\delta \tau=0.01$.

The convergent nature of the procedure is assessed by displaying successive estimates of the absolute growth rate $\omega_{0, i}(m=1)$ (figure $3 a$ ) and frequency $\omega_{0, r}(m=1$ ) (figure $3 b$ ) as a function of $\tau_{n}$. The three choices for $t_{2}$ are distinguished by 

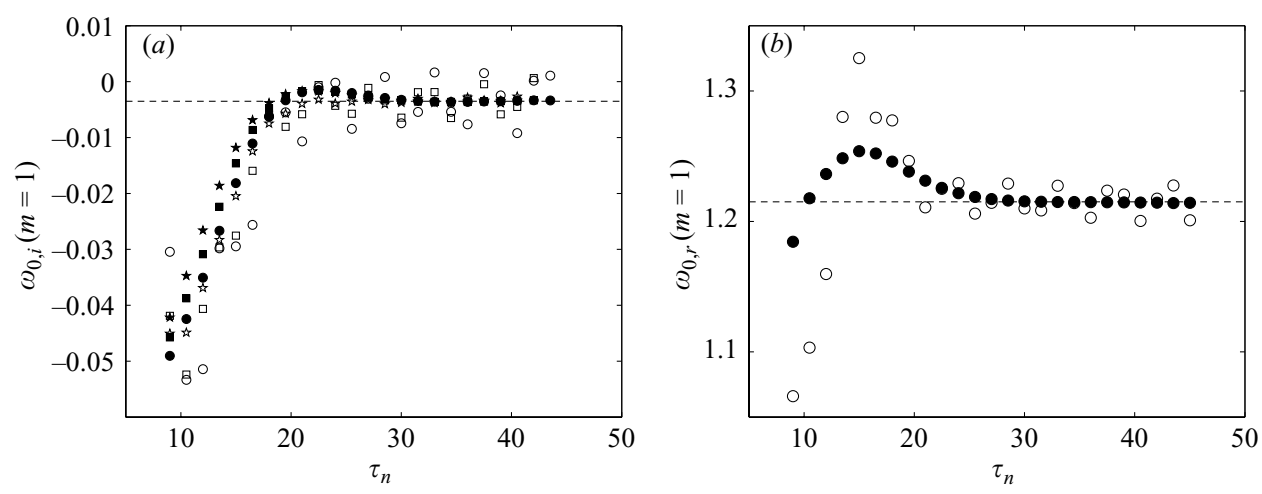

Figure 3. Convergence properties of the absolute frequency $\omega_{0}(m=1)$ as a function of discrete time $\tau_{n}$ for $\operatorname{Re}=200, S=1$ and $z=4.6:(a)$ absolute growth rate $\omega_{0, i}$ and $(b)$ frequency $\omega_{0, r}$. (a) Formula (3.5) with $t_{1}=\tau_{n-1}$ and $t_{2}=\tau_{n}(\bigcirc), \tau_{n+1}(\square), \tau_{n+2}(\star)$. Hollow symbols: Hilbert transform method; solid symbols: Fourier transform method.

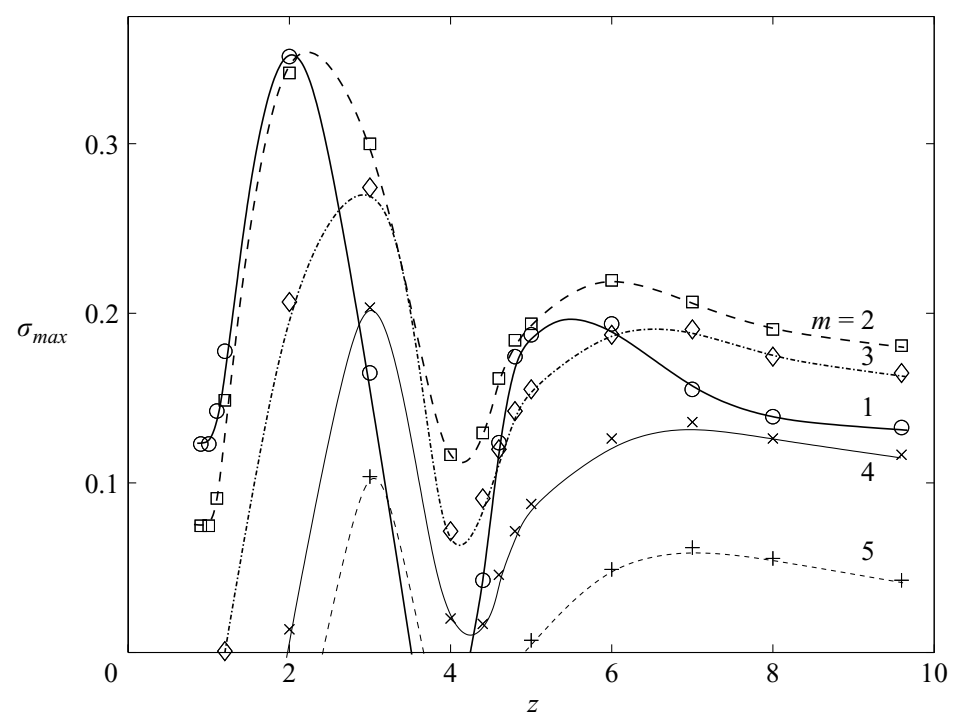

FIGURE 4. Streamwise evolution of the maximum temporal growth rate over all axial wavenumbers for various azimuthal modes $m=1,2,3,4,5$ at $R e=200$ and $S=1$.

different symbols, as detailed in the figure caption. Hollow symbols are obtained via Hilbert transform and solid symbols via the usual Fourier transform. The asymptotic convergence of the estimates for $\omega_{0, i}$ and $\omega_{0, r}$ is clearly apparent, justifying the entire procedure.

\section{Comparison between DNS results and predictions of nonlinear global mode theory}

The above diagnostic tools may now be applied at different streamwise stations in order to evaluate the relevance of nonlinear global mode concepts, as introduced for instance by Pier et al. (2001). A synthetic view of local temporal instability properties is obtained by displaying the streamwise evolution of the maximum temporal growth rate (over all axial wavenumbers) for various azimuthal wavenumbers $m=1 \ldots 5$ (figure 4). Higher-order azimuthal modes have been checked to remain stable. The 


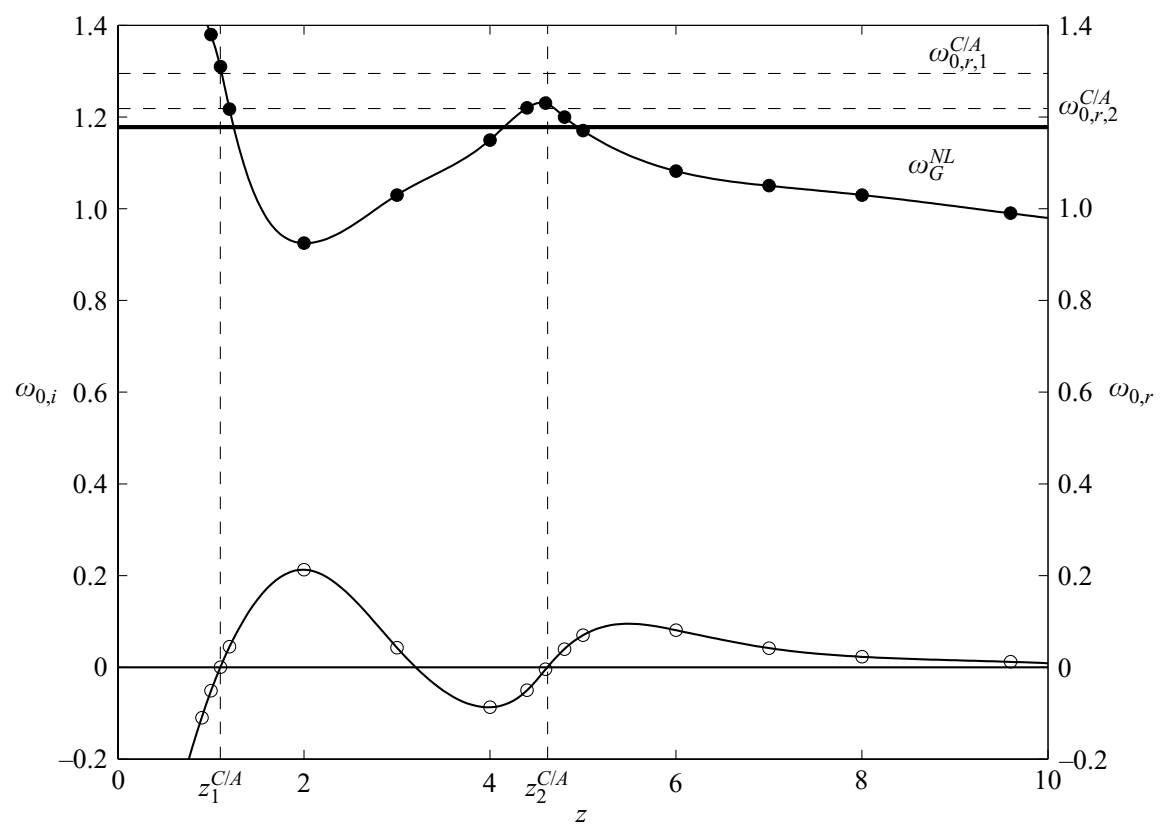

FIGURE 5. Streamwise evolution of the real part $\omega_{0, r}$ of the local absolute frequency (upper curve, right axis) and imaginary part $\omega_{0, i}$ (lower curve, left axis) for mode $m=1$ as a function of the streamwise coordinate $z$ for $R e=200$ and $S=1$. The predicted real absolute frequencies $\omega_{0,1}^{C / A}$ and $\omega_{0,2}^{C / A}$ at the $\mathrm{C} / \mathrm{A}$ transition points are indicated by dashed horizontal lines whereas the 'measured' global frequency $\omega_{G}^{N L}$ is marked by a solid horizontal line.

most unstable mode is observed to be $m=1$ in the upstream part of the flow $(z<2)$ and $m=2$ further downstream $(z>2)$. Temporal stability clearly does not succeed in yielding a sharp mode selection criterion accounting for the observed prevalence of the $m=1$ spiral mode $(\S 2)$.

The local absolutely/convectively unstable nature of the axisymmetric vortex breakdown state may be summarized by depicting the streamwise evolution of the absolute growth rate $\omega_{0, i}(m)$ and frequency $\omega_{0, r}(m)$ as a function of $z$ for different values of $m$. The case of the $m=1$ mode is displayed in figure 5 for the reference case $R e=200$ and $S=1$. Two absolutely unstable regions where $\omega_{0, i}>0$ are clearly in evidence: a first zone coinciding with the recirculation bubble located between $z_{1}^{C / A}=1.1$ and $z_{1}^{A / C}=3.3$ and a second zone in the lee of the bubble for $z$ larger than $z_{2}^{C / A}=4.7$. The occurrence of the first region is not unexpected. The presence of counterflow in the recirculation bubble is known to promote absolute instability. The existence of a less intense downstream region of absolute instability is in accordance with the qualitative results of Ruith et al. (2003): the local base flow pertaining to axisymmetric breakdown was indeed shown to enter the absolutely unstable domain twice when approximately modelled by a Batchelor vortex.

According to the theoretical analysis of Pier et al. (2001) the observed global frequency should be given by the real absolute frequency $\omega_{0, r}^{C / A}$. In view of figure 5, two candidate stations are in competition with associated frequencies $\omega_{0,1}^{C / A}=1.30$ and $\omega_{0,2}^{C / A}=1.22$ represented by dashed horizontal lines. The effective global frequency $\omega_{G}^{N L}=1.18$ measured in the DNS is shown by a solid horizontal line in figure 5 . Comparison with the previous values suggests that the downstream transition point $z_{2}^{C / A}$ may be the wavemaker responsible for the flow synchronization. A similar 


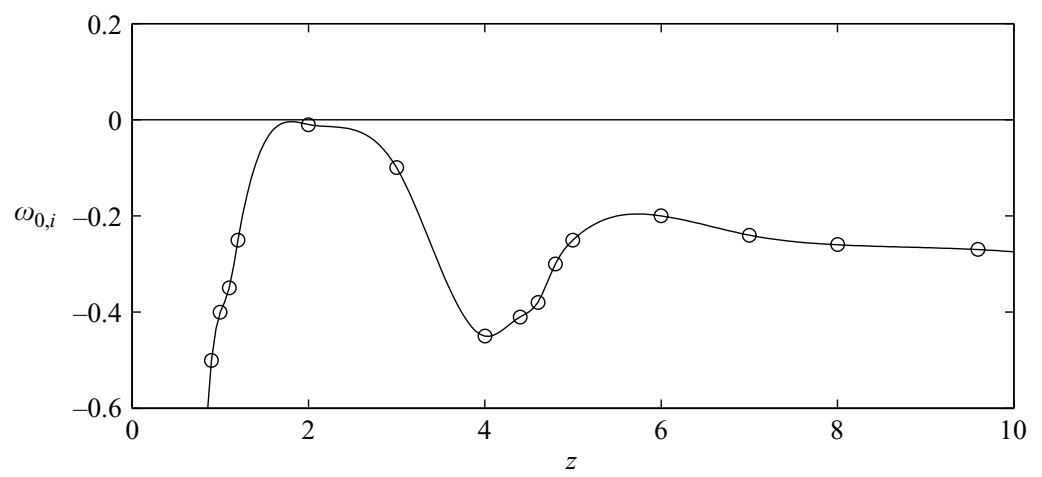

FIGURE 6. Streamwise evolution of the imaginary part $\left(\omega_{0, i}\right)$ of the local absolute frequency for mode $m=2$ as a function of the streamwise coordinate $z$ for $R e=200$ and $S=1$.

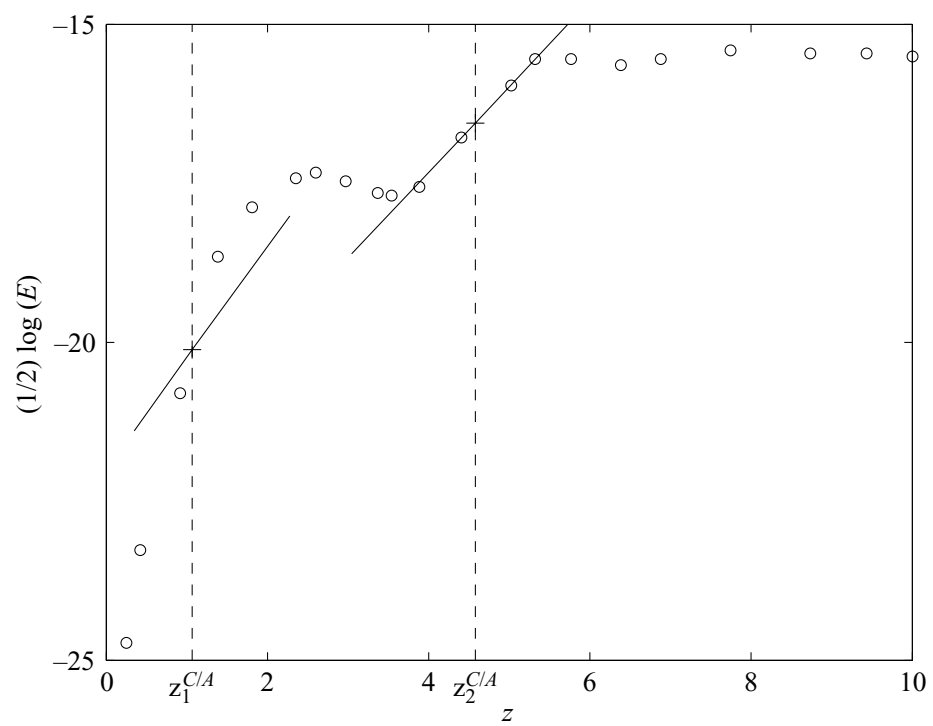

FIGURE 7. Perturbation amplitude distribution as a function of streamwise coordinate $z$ for $R e=200$ and $S=1$. The two spatial absolute growth rates $-k_{0, i, 1}^{C / A}$ and $-k_{0, i, 2}^{C / A}$ are represented by straight lines located at the corresponding $\mathrm{C} / \mathrm{A}$ transition point.

analysis may be carried out for higher values of $m$. For instance the variations of $\omega_{0, i}(m=2)$ are displayed in figure 6 . The helical mode $m=2$ is seen to remain convectively unstable at all axial stations. The same behaviour holds for larger $m$. The validity of the interpretation in terms of a nonlinear global mode with a front located at the second transition point $z_{2}^{C / A}$ may be further ascertained by considering the streamwise amplitude distribution $\sqrt{E(z)}$ at $t=1850$ (figure 7) extracted from the DNS, where the energy $E(z)$ is defined as

$$
E(z)=\int_{0}^{2 \pi} \int_{0}^{R} \frac{1}{2}\left(u_{z}^{\prime 2}+u_{r}^{\prime 2}+u_{\theta}^{\prime 2}\right) r \mathrm{~d} \theta \mathrm{d} r .
$$

In the above expression, $u_{z}^{\prime}, u_{r}^{\prime}$ and $u_{\theta}^{\prime}$ denote the observed nonlinear perturbations from the axisymmetric breakdown state. The disturbance amplitude isseen to grow 
in two successive stages: the first one occurs near $z_{1}^{C / A}$, the second near $z_{2}^{C / A}$. More strikingly, the slope of the amplitude curve at $z_{2}^{C / A}$ coincides with $-k_{0, i, 2}^{C / A}$, i.e. the spatial absolute growth rate at $z_{2}^{C / A}$. This feature is in full agreement with the steep global mode theory of Pier et al. (2001): the linearly selected front at $z_{2}^{C / A}$ should grow exponentially with the aforementioned spatial growth rate. Note also that the slope of the amplitude curve at $z_{1}^{C / A}$ is not given by $-k_{0, i, 1}^{C / A}$. In other words, the flow selects a nonlinear global mode with a front located at $z_{2}^{C / A}$.

The above findings have been further confirmed by performing a similar analysis at a higher swirl number $S=1.095$ and at the same Reynolds number $R e=200$. The axisymmetric breakdown state still displays two absolutely unstable regions and the second transition point predicts a value $\omega_{0,2}^{C / A}=1.26$ to be compared with the 'measured' value $\omega_{G}^{N L}=1.20$.

\section{Discussion and conclusions}

According to the above analyses, spiral vortex breakdown may be interpreted as a nonlinear global mode which develops on the axisymmetric breakdown state. It is triggered by the appearance of a locally absolutely unstable region in the wake of the breakdown bubble. The nonlinear global mode is primarily driven by a front located at the convective to absolute instability transition station. As in steep global mode theory, the nonlinear global frequency is thereby given by the absolute frequency $\omega_{0,2}^{C / A}$ at the transition station and the front slope coincides with the absolute spatial growth rate $-k_{0, i, 2}^{C / A}$. In other words, the breakdown bubble is analogous to a bluff body, the wake of which becomes absolutely unstable. In both instances, self-sustained oscillations arise in the form of a nonlinear global mode and the frequency selection mechanisms are identical (see Pier \& Huerre 2001). Note again that the weakly nonparallel assumption is clearly violated by the base flow (figure 2). Furthermore the absolute wavenumber $k_{0, r}=0.48$ corresponds to a wavelength $\lambda_{0}=13.1$. This is not small compared to the streamwise inhomogeneity length scale. But it is consistent with the DNS results (see figure $1 b$ ).

It remains to understand the passive role of the absolutely unstable vortex breakdown bubble which does not seem to partake in the frequency selection. Currently available weakly non-parallel formulations are incapable of treating such a strongly non parallel object. We can only speculate that, as in the linear setting (Chomaz, Huerre \& Redekopp 1991), the absolutely unstable region is too small to be active. Note however that Liang \& Maxworthy (2005) and Billant et al. (1998) have experimentally observed spiral vortex breakdown being initiated within the recirculation bubble. This phenomenon occurs at a different inlet swirl number and for a different inlet velocity profile.

Another issue concerns higher-order helical modes which are known to arise at higher swirl and Reynolds numbers (Ruith et al. 2003). Understanding this process may involve examining the competition between distinct incipient global modes.

François Gallaire and Michael Ruith gratefully acknowledge the financial support of Ecole Polytechnique. All computations have been performed at IDRIS-CNRS.

\section{REFERENCES}

BERS, A. 1983 Space-time evolution of plasma instabilities-absolute and convective. In Handbook of Plasma Physics (ed. M. Rosenbluth \& R. Sagdeev), pp. 451-517. North-Holland. 
Billant, P., Chomaz, J.-M. \& Huerre, P. 1998 Experimental study of vortex breakdown in swirling jets. J. Fluid Mech. 376, 183-219.

BrüCKer, C. \& Althaus, W. 1995 Study of vortex breakdown by Particle Tracking Velocimetry (PTV). Part 3: Time-dependent structure and development of breakdown modes. Exps. Fluids 18, $174-186$.

Chomaz, J.-M. 2005 Global instabilities in spatially developing flows: non-normality and nonlinearity. Annu. Rev. Fluid Mech. 37, 357-392.

Chomaz, J.-M., Huerre, P. \& Redekopp, L. G. 1991 A frequency selection criterion in spatially developing flows. Stud. Appl. Maths 84, 119-144.

Couniron, A. \& Chomaz, J.-M. 1999 Fully nonlinear global modes in slowly varying flows. Phys. Fluids 11, 3688-3703.

Delbende, I., Chomaz, J.-M. \& Huerre, P. 1998 Absolute/convective instabilities in the Batchelor vortex: a numerical study of the linear impulse response. J. Fluid Mech. 355, 229-254.

Escudier, M. P. 1988 Vortex breakdown : observations and explanations. Prog. Aerospace Sci. 25, 189-229.

Escudier, M., Bornstein, J. \& Maxworthy, T. 1982 The dynamics of confined vortices. Proc. R. Soc. Lond. A 382, 335-360.

Escudier, M. \& Zehnder, N. 1982 Vortex-flow regimes. J. Fluid Mech. 115, 105-121.

FAler, J. \& Leibovich, S. 1977 Disrupted states of vortex flow and vortex breakdown. Phys. Fluids 20, 1385-1400.

Gallaire, F. \& Chomaz, J.-M. 2003 Mode selection in swirling jet experiments: a linear stability analysis. J. Fluid Mech 494, 223-253.

Grabowski, W. \& Berger, S. 1976 Solutions of the Navier-Stokes equations for vortex breakdown. J. Fluid Mech. 75, 535-544.

Huerre, P. \& Monkewitz, P. A. 1990 Local and global instabilities in spatially developing flows. Annu. Rev. Fluid Mech. 22, 473-537.

LAmbourne, N. C. \& Bryer, D. W. 1961 The bursting of leading-edge vortices: some observations and discussion of the phenomenon. Aero. Res. Counc. $R \& M$ 3282, 1-35.

Liang, H. \& Maxworthy, T. 2005 An experimental investigation of swirling jets. J. Fluid Mech. 525, 115-159.

Pier, B. \& Huerre, P. 2001 Nonlinear self-sustained structures and fronts in spatially developing wake flows. J. Fluid Mech. 435, 145-174.

Pier, B., Huerre, P. \& Chomaz, J.-M. 2001 Bifurcation to fully nonlinear synchronized structures in slowly varying media. Physica D 148, 49-96.

Ruith, M., Chen, P., Meiburg, E. \& Maxworthy, T. 2003 Three-dimensional vortex breakdown in swirling jets and wakes: direct numerical simulation. J. Fluid Mech. 486, 331-378.

SARPKAYA, T. 1971 On stationary and travelling vortex breakdown. J. Fluid Mech. 45, 545-559.

Spall, R. E. \& GATSKI, T. B. 1991 A computational study of the topology of vortex breadkown. Proc. R. Soc. Lond. A 435, 321-337.

Yin, X., Sun, D., WEI, M. \& WU, J. 2000 Absolute and convective instability character of slender viscous vortices. Phys. Fluids 12, 1062-1072. 